\title{
نشأة متاحف الفن بالجزائر خلال الفترة الفرنسية
}

The emergence of art museums in Algeria during the French period

slus

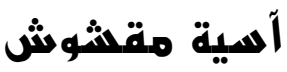

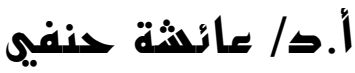

استاذة التعليم العالي معهد علم الاثار - الجز ائر

Doi: 10.21608/kjao.2020.116866

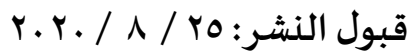

استلام البحث : Y. r. / V / Y

المستخلص:

عرفت الجزائر في بدايات الاحتلال الفرنسي استقبال عدد كبير من الفنانين، الذين كانوا مولعين بسحر الجزائر من عمارة إسلامية، و لباس و عادات وتقاليد جعلت مخيلة الفنان الأوربي خصبة لإنتاج عدد جد مهم من اللوحات المحفوظة اليوم بمتاحف الجزائر، ومتاحف العالم ،وباستقرار المستعمر لمدة قرن وثلاثثن سنة استقر الفن في الجزائر وأسس لنفسه مدرسة جديدة عرفة بالمدرسة الجزائرية، رسمت واقع الجزائر ولم ترسم مخيلة الفنان

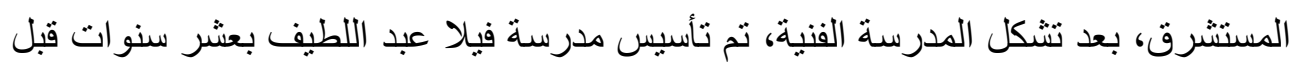
احتفالية المئة سنة على احتلال الجزائر، خلال هذه المدة تضخم رصيد المتحف البلدي الذي كان يحفظ مجموعة من الأعمال الفنية، ولم يصبح قادرا على استيعاب المجموعة كاملة، فتم تتيييد متحف الفنون الجميلة بالجزائر سنة ـ بوا بهذه المناسبة، وتأسيس متحف سيرتا ومتحف أحمد زبانة، كمناحف جهوية لحفظ الأعمال الفنية. الكلمات المفتاحية: متحف، عمارة المتحف، الفنون الجميلة، فيلا عبد اللطيف،المستثرقون،

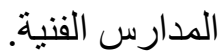




\section{Abstract:}

Algeria knew at the beginning of the French occupation, the reception of a large number of artists, who were fond of the magic of Algeria from an Islamic architecture, and the clothing, customs and traditions that made the imagination of the European artist fertile for the production of a very important number of paintings preserved today in the museums of Algeria, the museums of the world, and the stability of the colonist for a century and thirty years Art settled in Algeria and established for itself a new school in the Algerian school, which drew the reality of Algeria and did not paint the imagination of the oriental artist. After the art school was formed, the Villa Abdul Latif School was established ten years before the celebration of the 100 years since the occupation of Algeria, during this period the balance of the municipal museum that He was saving a collection of artworks, and he was not able to absorb the whole collection. The Museum of Fine Arts in Algeria was built in 1930 on this occasion, and the Cirta Museum and Ahmed Zabana Museum were established as regional museums for preserving artwork.

key words: Museum, museum architecture, fine arts, villa Abdullatif, orientalists 'art schools 
لم تعرف الجزائر الفنون التشكيلية في شكلها الغربي، قبل الاحتلال الفرنسي، بل كانت

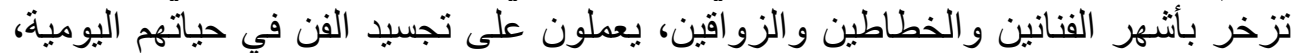

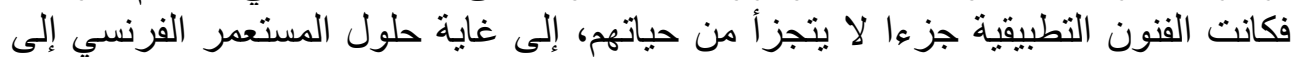

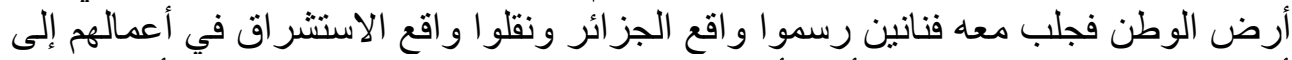

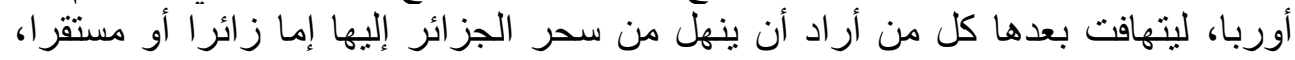

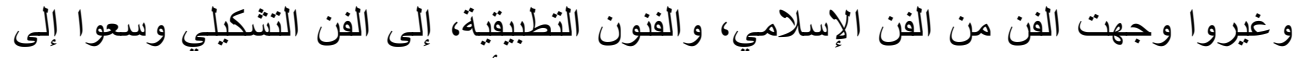

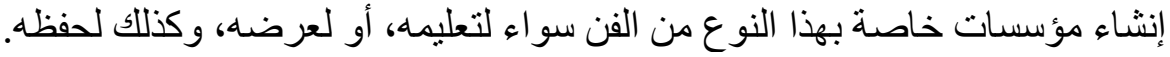

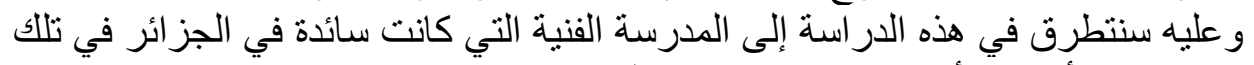

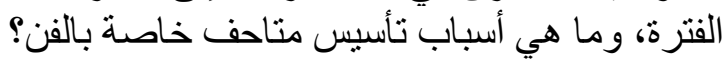

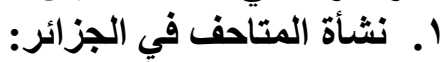

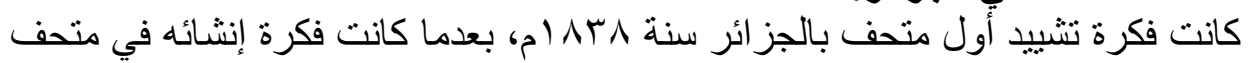

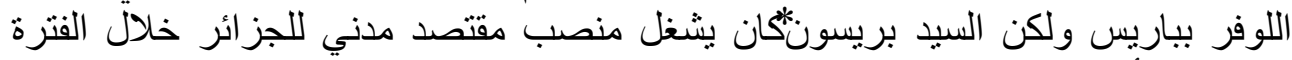

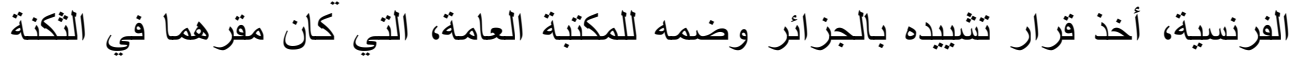

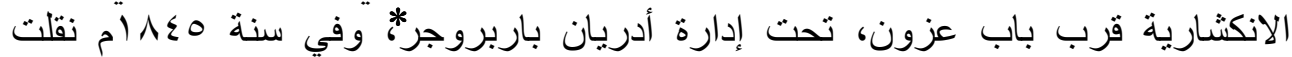

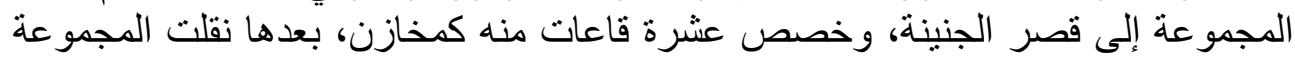

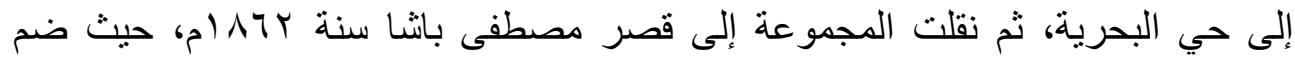

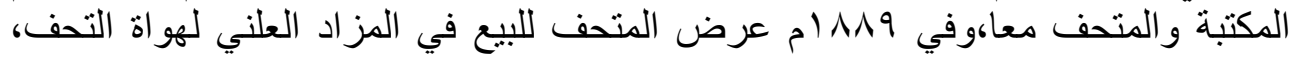
وبعد تعيين.

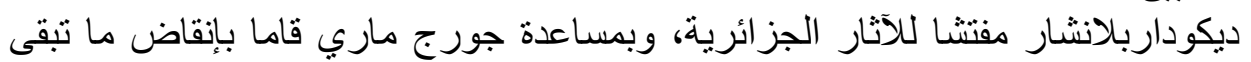

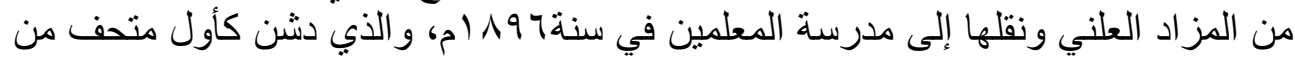

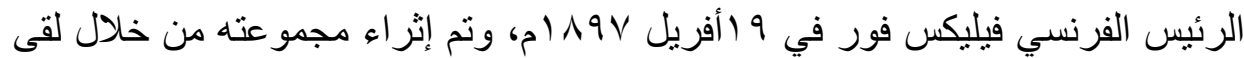

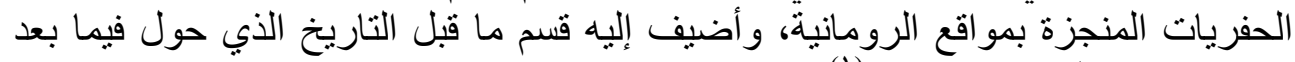

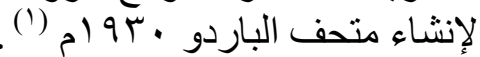
بعد سنة من تأسيس جمعية الآثار لمقاطعة قسنطينة، جمع أعضاء الأناء الجمعية كل اللقى

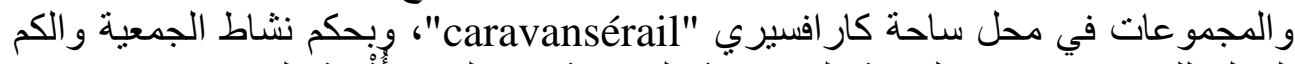

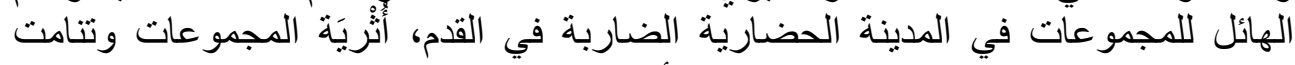

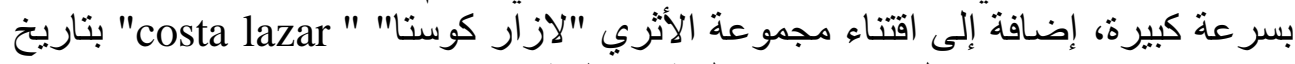

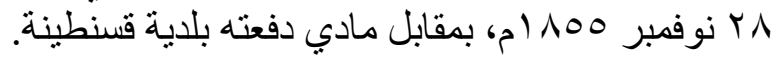




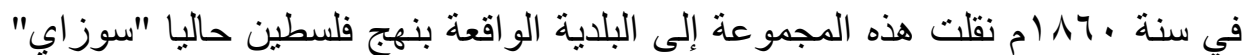

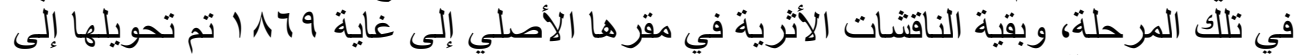
الحديقة العامة (؟) الحرة

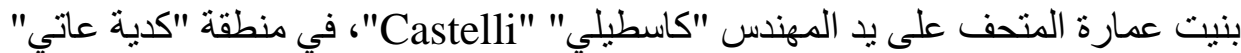

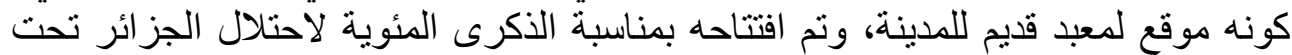

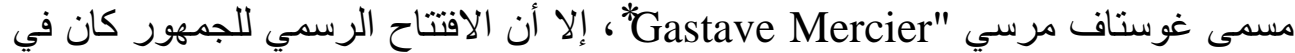

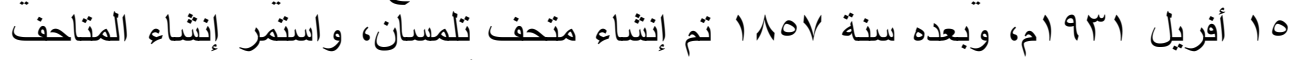

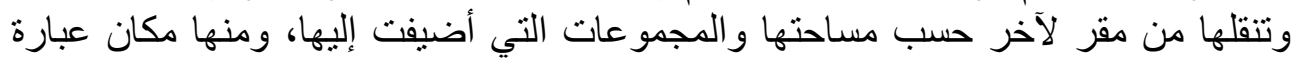

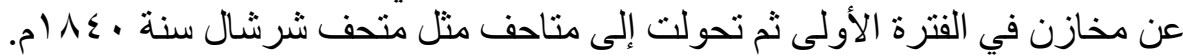

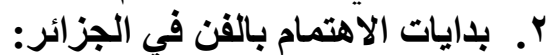

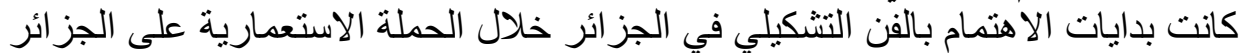

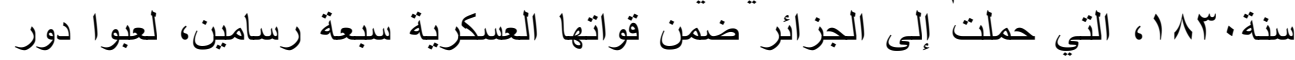

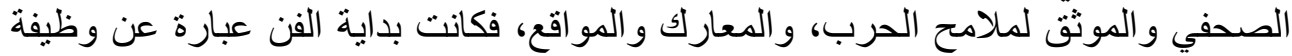

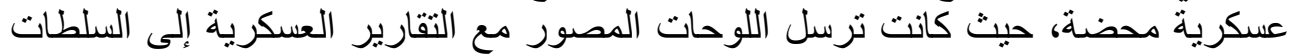

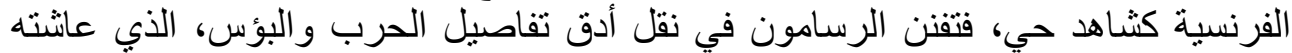

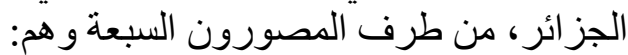

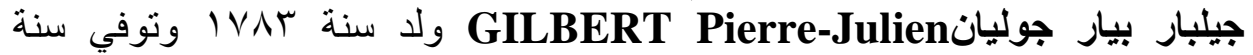
• 1 ا م، شغل منصب معلم للرسم في البحرية الملكية الفرنسية، و عليه تم تعبين قائدا للفرقة

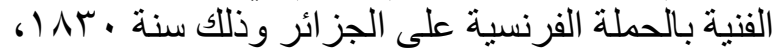

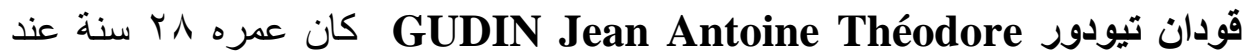
تعيينه مر افقا فنيا للقوات العسكري يحفظ له متحف الفنون الجميلة الجز ائر أربعة أعمال هي Cote de ".. "Inauguration de la chapelleb " Mort de st-Louis 1270 " IG 350, IG 351, ) تحت أرقام جرد " Presqu' ile de sidi-Ferruch " "barbarie

(IG352IG353,

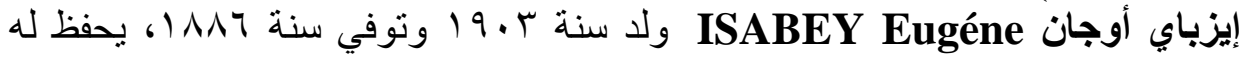

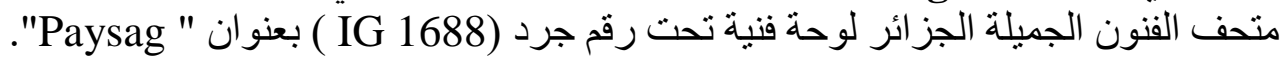

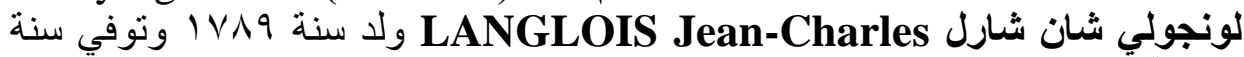

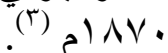

MOREL و تورني دي بالفيل TOURNIE De Belleville (؛ و فُشموت فرديناند Fation

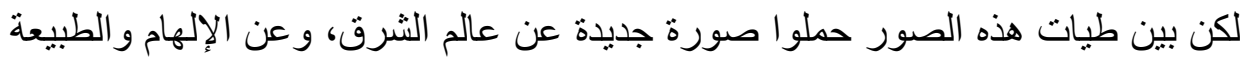

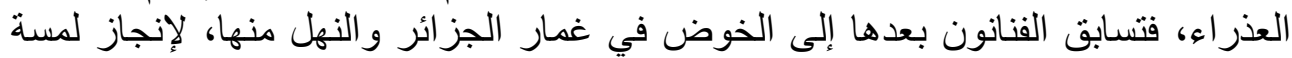




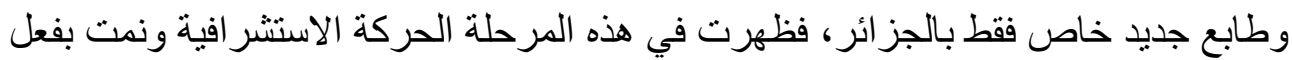

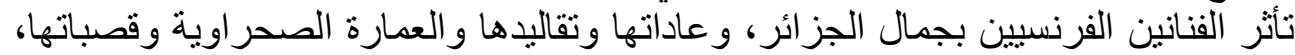

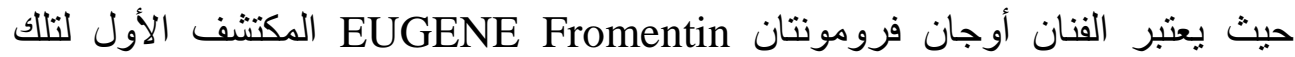

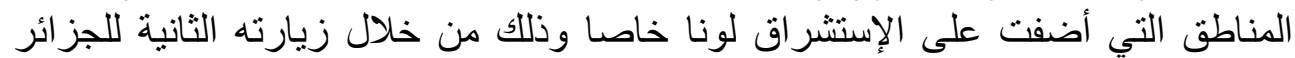

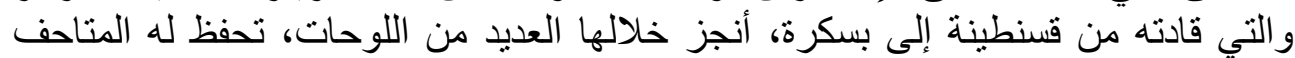

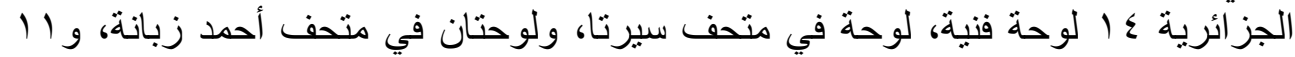

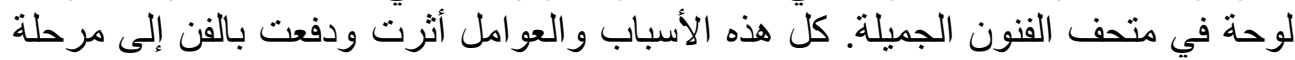

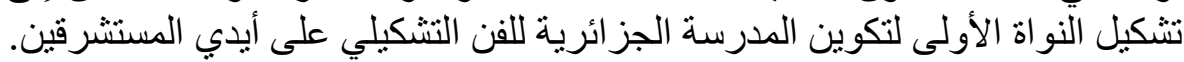

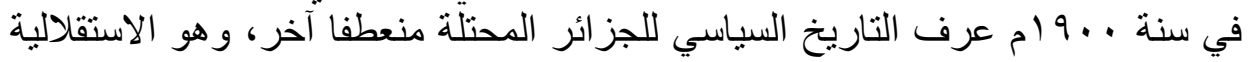

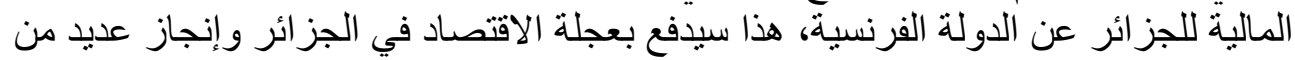

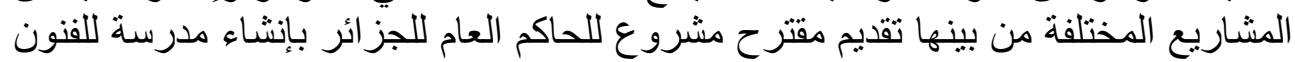

التشكيلية.

r. إنشاء مدرسة فيلا عبد اللطيف: تقع في منطقة منحدر المدنية، بأعالي العاصمة ،نطل من من الناحية الثمالية على متحف

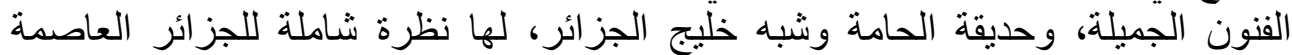

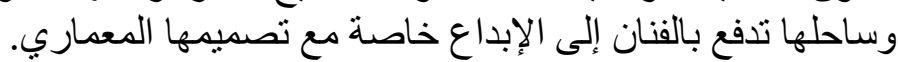

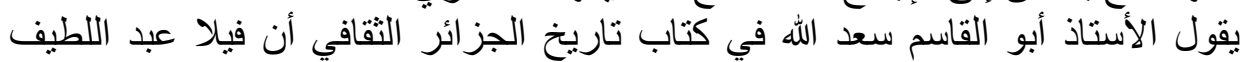

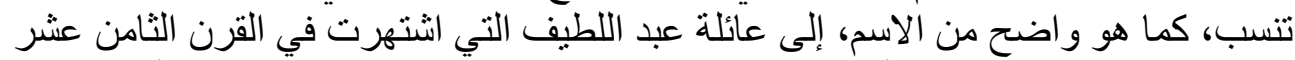

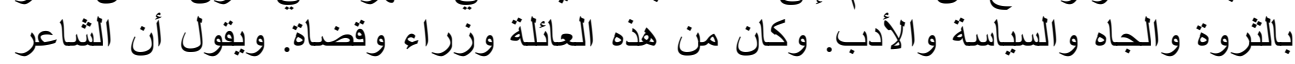

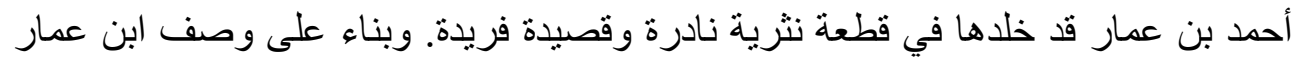

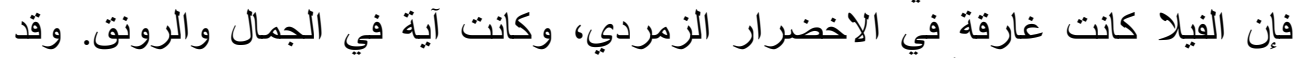

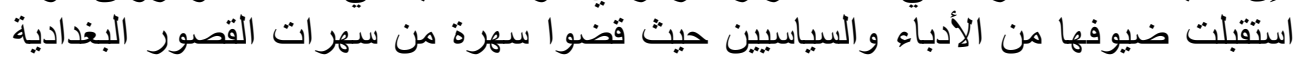
والأندلسية. (0) فئن

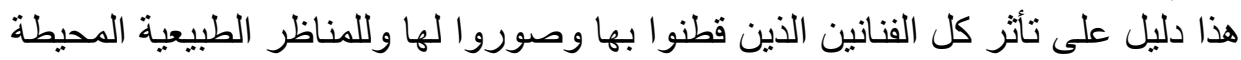

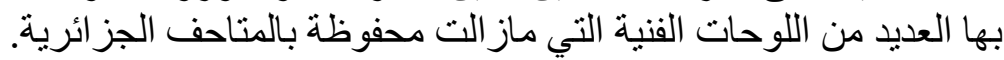

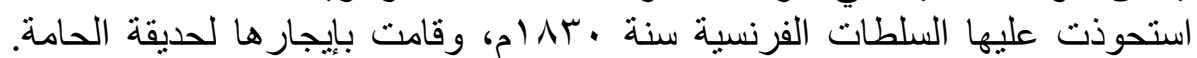
وفي سنة V.9 (1، اصدر الحاكم العام للجزائر قرارا بتحويلها إلى مدرسة للفنون التشكيلية

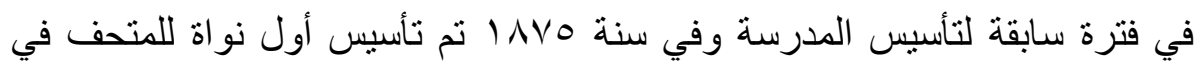

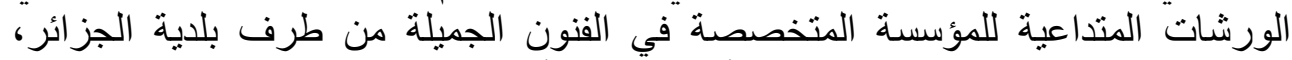

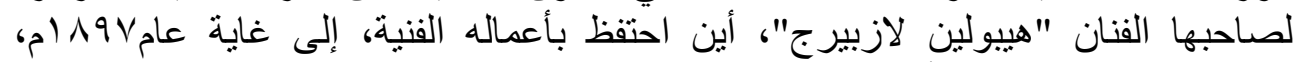
وجمعت فيه مجموعة من أعمال الفن القديم والفنون الإسلامية، وبعد إخلاء ثكنة عسكرية تقع الفية عالفية 


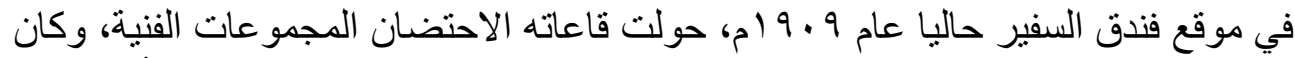

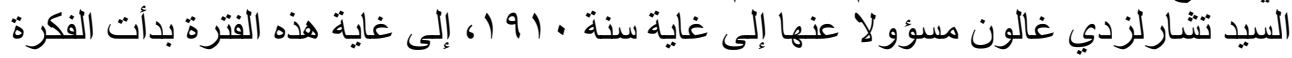

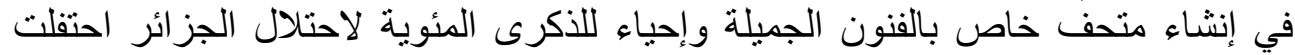

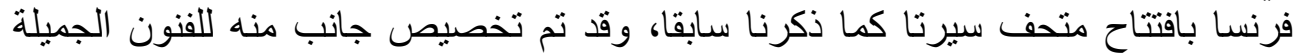

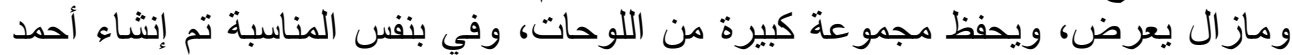

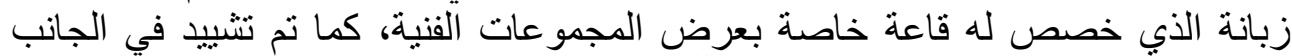

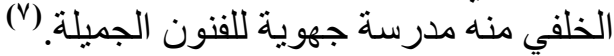

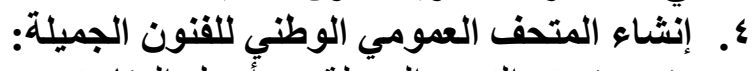

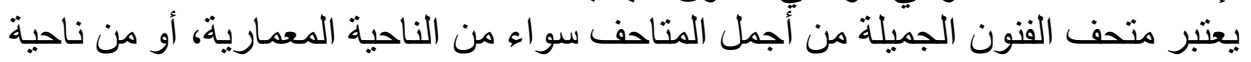

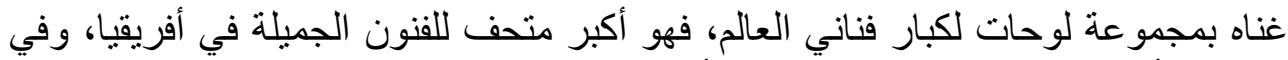

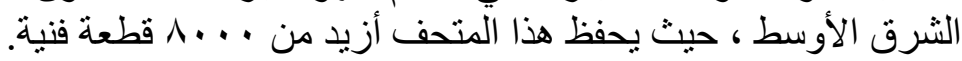

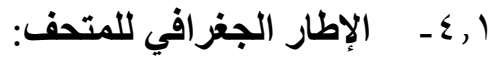

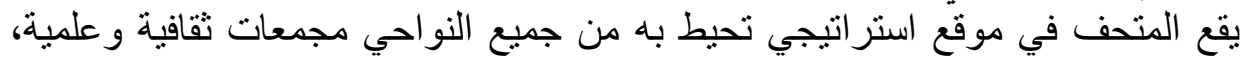

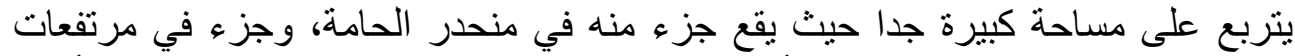

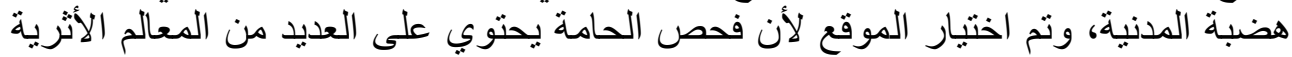

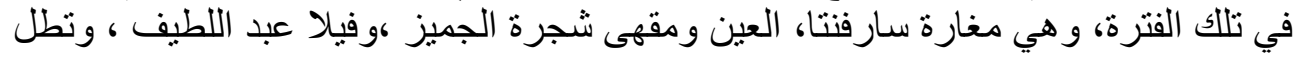

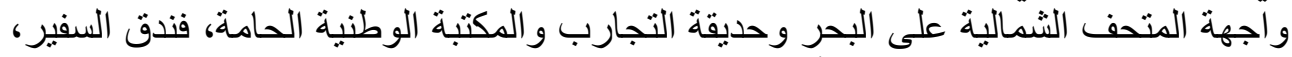

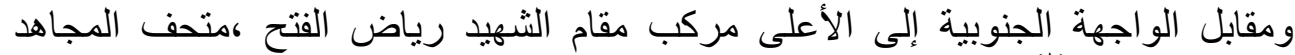

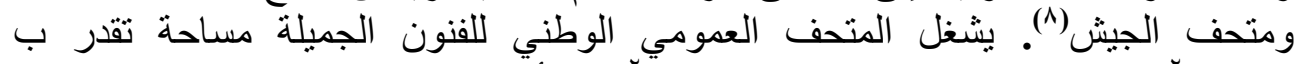

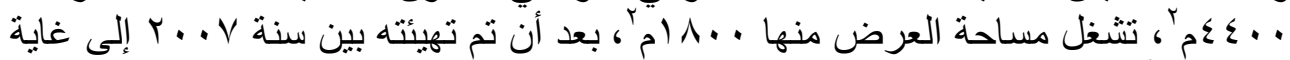

(9) ( $^{r} \cdot 1$.

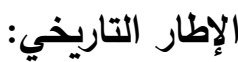

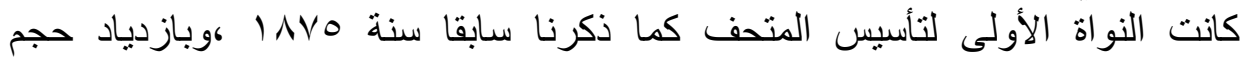

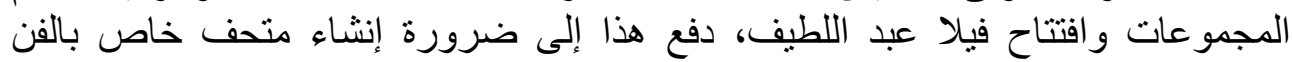

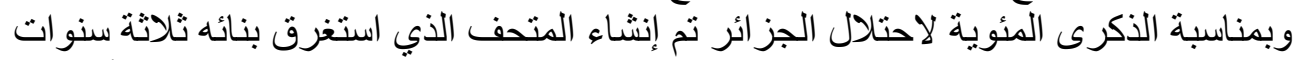

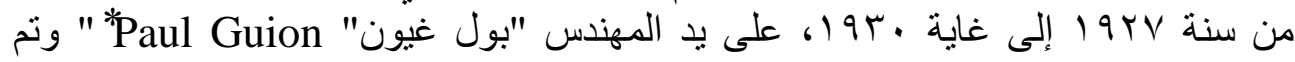

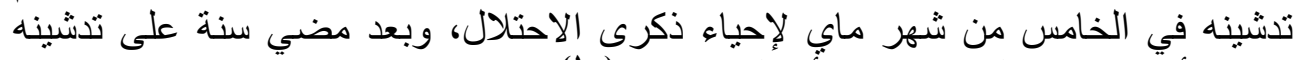

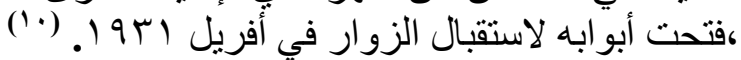

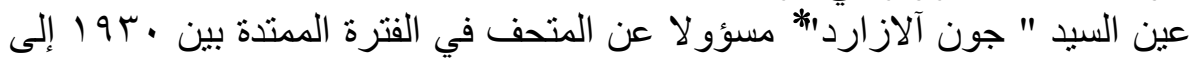

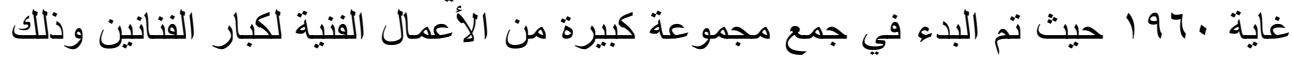

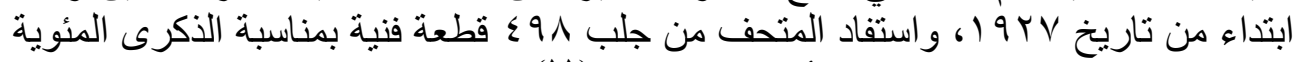

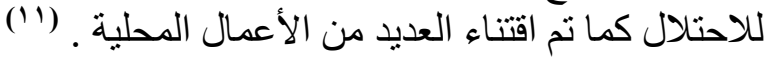


قبيل الاستقلال تم تفجير المتحف من طرف منظمة "الأقدام السوداء"، ونقلت الحكومة

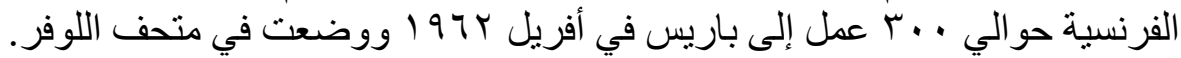

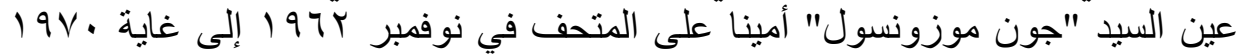

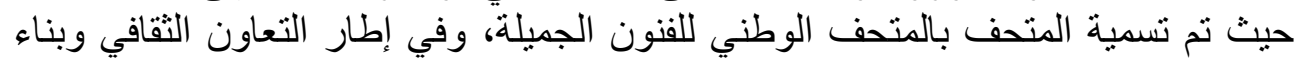

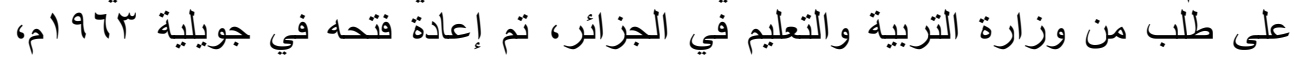

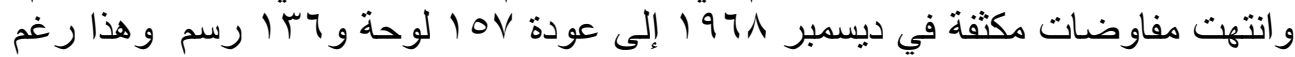

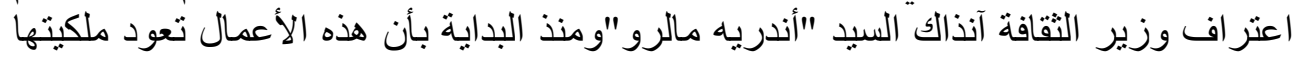

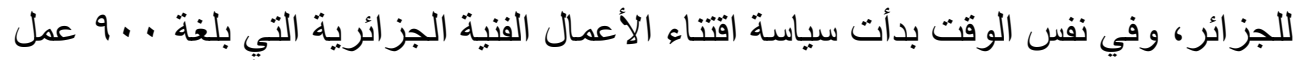

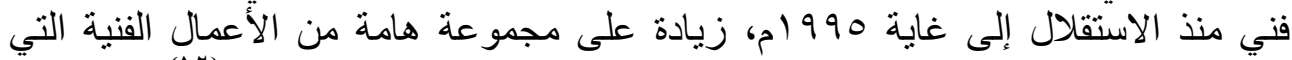

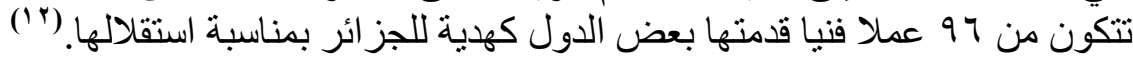

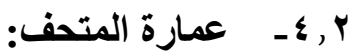
يتكون المبنى من عمارتين مدمجتين مع بعضهما البعض، البعض، عمارة تسبق الأخرى و العمارة

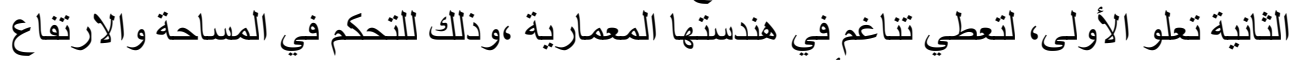

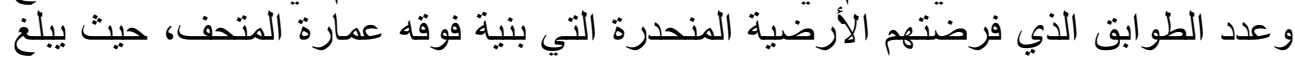

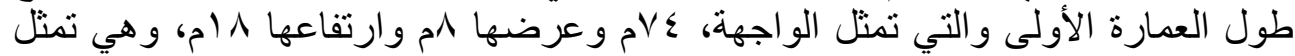

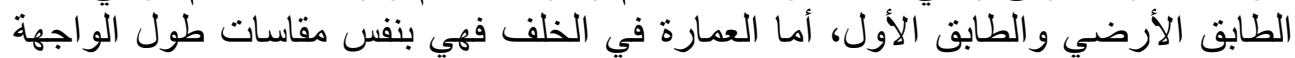

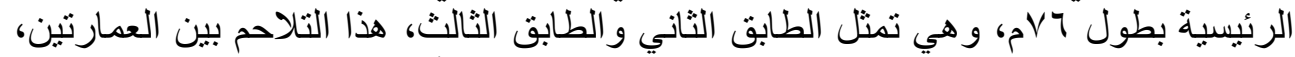

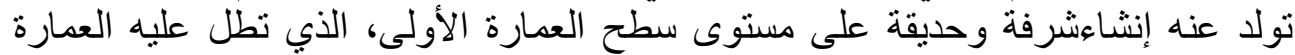

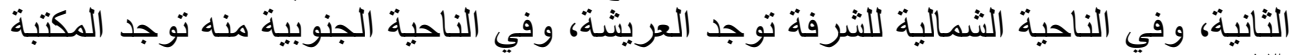

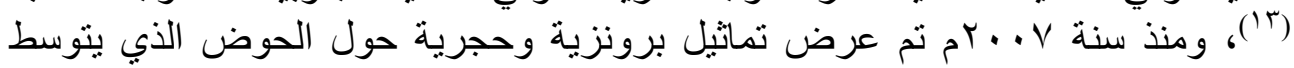
الحديقة وتحت الأعمدة الحاملة للعريشة. 

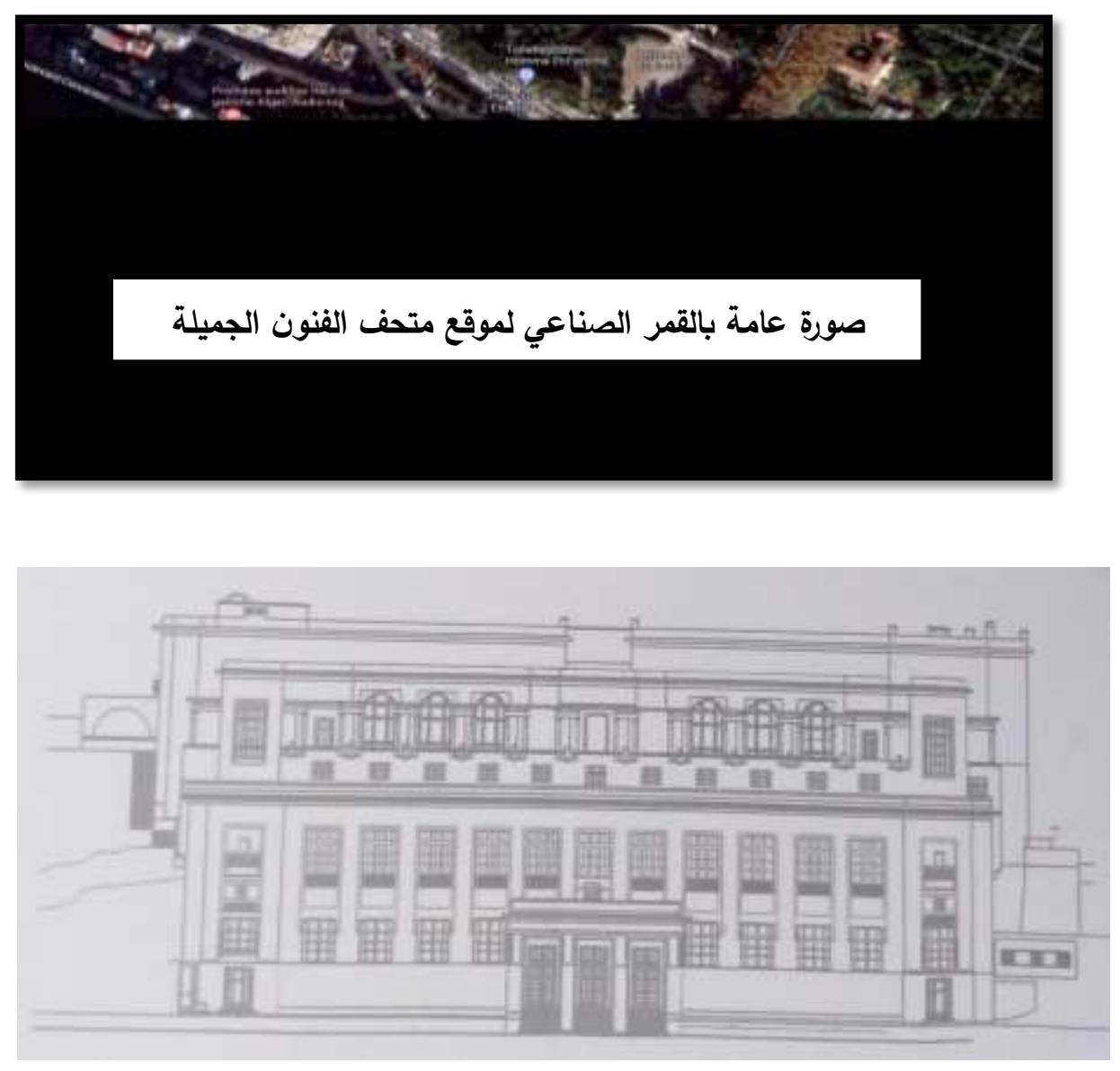

مخطط الواجهة الجنوبية لمتحف الفنون الجميلة 
أ. الطابق الأرضي والطابق الأول:

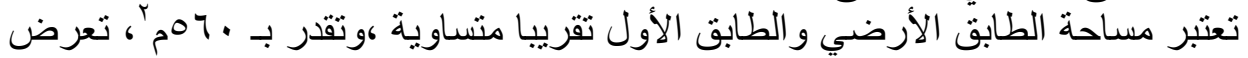

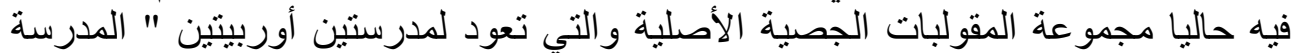

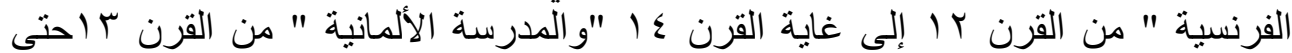

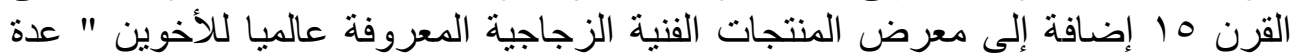

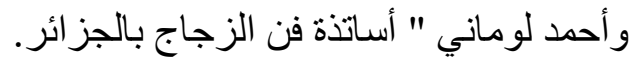

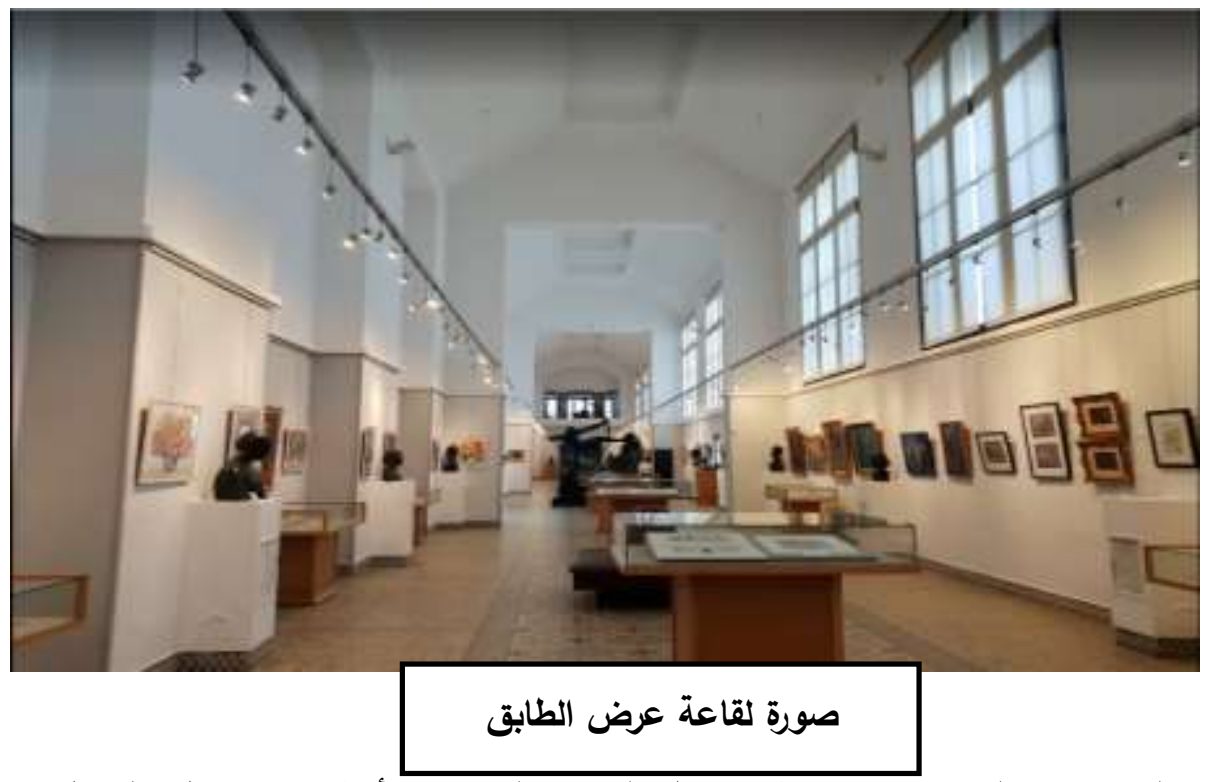

و المنحوتات البرونزية يتوسطها تمثال النحات الفرنسي "أنطوان بوردال" لتمثنال هرقل فئل

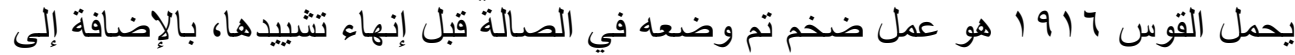

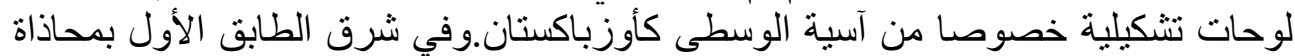

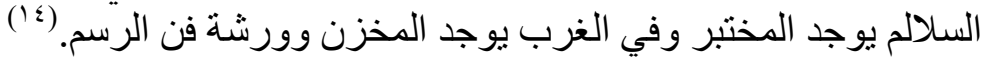

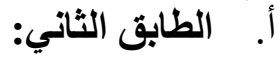

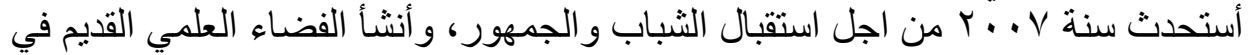

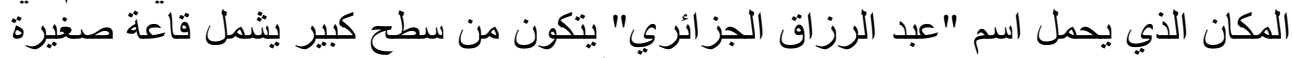
للمطالعة تتوفر على المنشورات الجديدة للفن، وأخيرا فضاء التهن للورنشات البيداغوجية ومقهى 


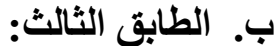

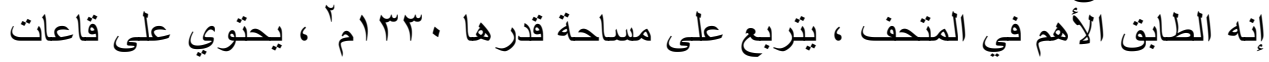

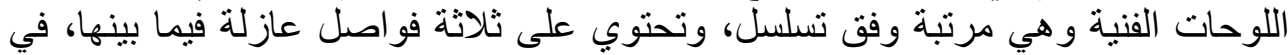

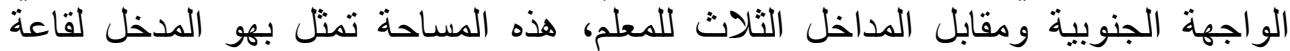

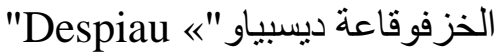

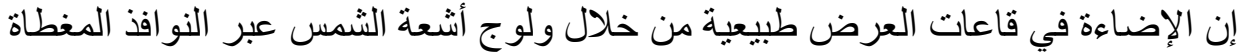

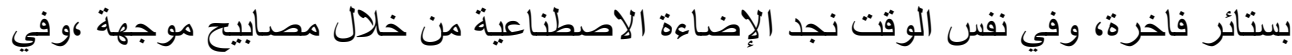

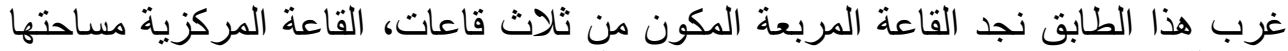

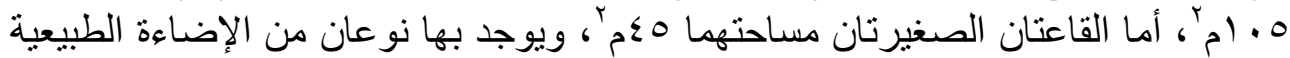

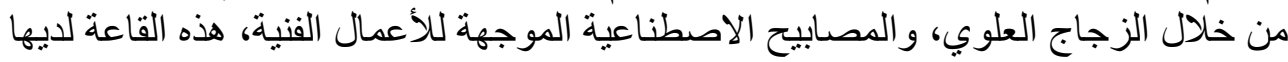

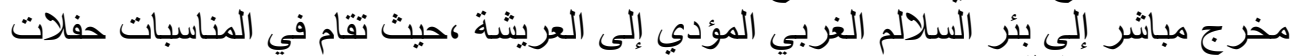
الغناء الأندلسي. (10)

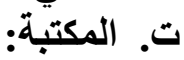

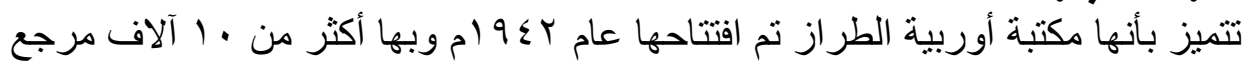

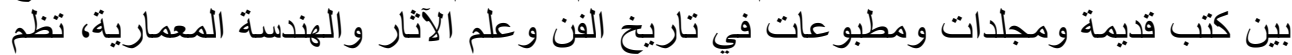

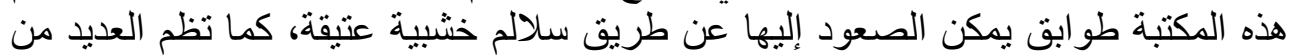
الأثاث الأصيل إضافة إلى أنها مزينة بلوحات للفنانة باية محي الدين. 


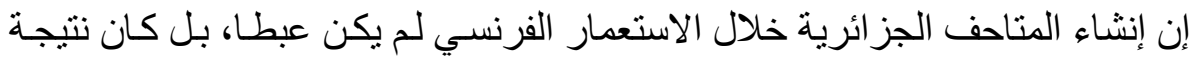

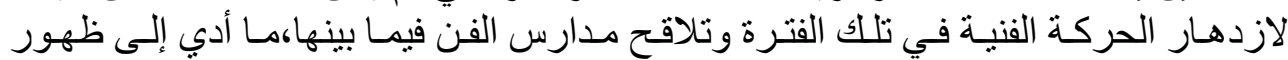

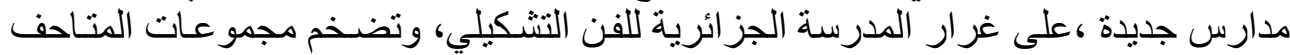

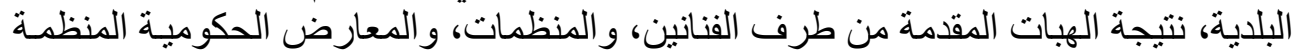

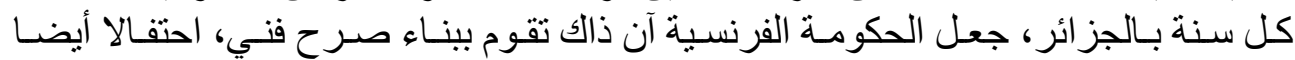

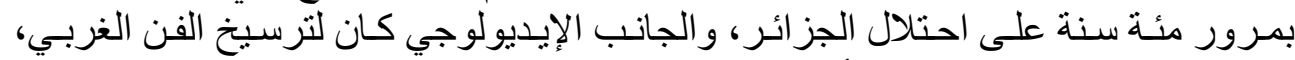

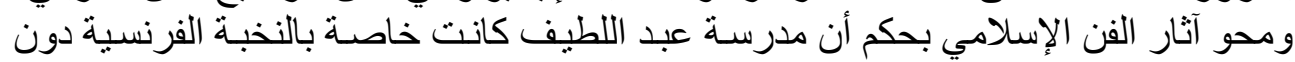

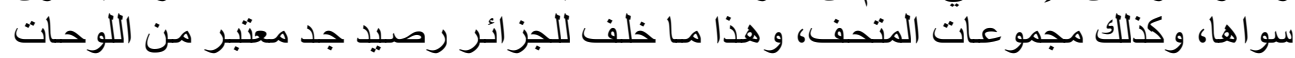

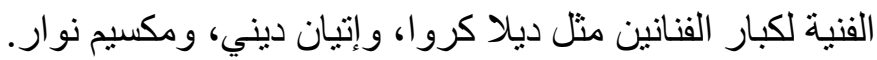


* * كان يشغل منصب مقتصد مدني للجز ائر خلال الفترة الفرنسية.

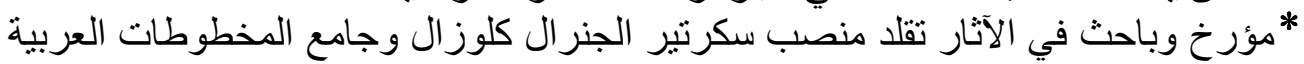

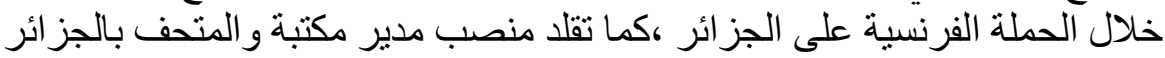

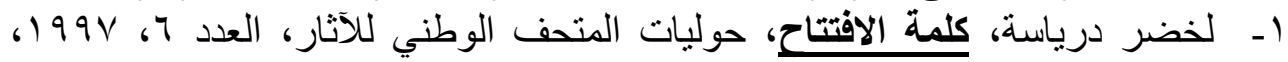

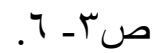

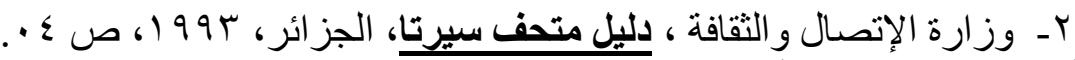

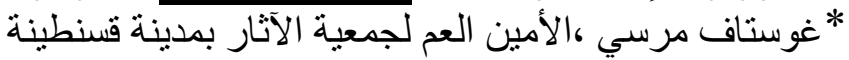

\section{3- DalilaMAHAMMED-ORFALI, Catalogue des peintures dessins} et gravures du musée national des baux art, Alger, 1995, p262. .4- Lila Fatima KHOUCHANE, Op.cit , p 04 * جون آلازارد عميد كلية الفنون الجميلة بالجزائر مختص في تاريخ الفن ،ومدير متحف 5- Dalila MAHAMMED-ORFALI,Op.cit , p10.

3 Ibid, p 16.

4 -Vidal Bué Marion, Alger et ses peintres 1830- 1960, Paris, 2000, P267

هـ أبو القاسم سعد الله، تاريخ الجزائر الثقافي، ج ^، ط ا، دار الغرب الإسلامي، 991996،

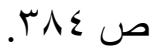

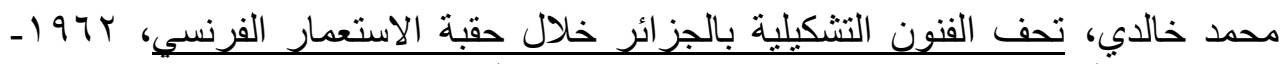

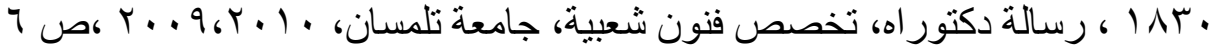

7 - Doumergue François, historique du musée d'Oran extrait du B.S.G.A.O. tome 54,1924 ,p68

8 -Lila Fatima KHOUCHANE, Musée National des Beaux-Arts Un joyau architectural et muséal, Alger, 2015, p 6.

9Ibid, $\mathrm{p} 14$.

$$
\text { *بول غيون مهندس ورسام ولد عام |AN| في مدينة قالمة. }
$$

10 -Lila Fatima KHOUCHANE, Op.cit , p 04.

* جون آلازارد عميد كلية الفنون الجميلة بالجزائر مختص في تاريخ الفن ،ومدير متحف

11Dalila MAHAMMED-ORFALI,Op.cit , p10.

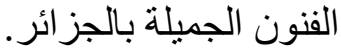


12 Ibid, p 16.

13Lila Fatima KHOUCHANE, Op.cit, p 14

14Lila Fatima KHOUCHANE, Op.cit ,p 14.

15Lila Fatima KHOUCHANE, Op.cit , p 10 
